\title{
Electrodeposition of Silver Amalgam on Thin Gold Film Electrodes for Voltammetric Detection of 4-nitrophenol and DNA labeled by osmium tetroxide-bipyridine complex
}

\section{$\underline{\text { Pavlina Havranova }}^{a}$,Filip Ligmajer ${ }^{a, b, c}$, Ales Danhel ${ }^{a *}$}

a Institute of Biophysics of the Czech Academy of Sciences, Kralovopolska 135, CZ-61265

Brno, Czech Republic

b Central European Institute of Technology, Brno University of Technology, Purkynova 123, CZ-612 00 Brno, Czech Republic

c Institute of Physical Engineering, Brno University of Technology, Technicka 2, CZ-616 69

Brno, Czech Republic

e-mail: danhel@ibp.cz

\begin{abstract}
Alternative electrode materials suitable for preparation of novel working electrode architectures applicable in detection of biopolymers such as nucleic acids, proteins or glycoproteins, represent a significant contribution in bio-electroanalysis. Herein, electrodes made of vapor-deposited gold thin films (vAuE) were used as alternative substrate for the electrodeposition of silver amalgam particles (AgAPs), next to already used indium tin oxide and pyrolytic graphite. Conditions and parameters of double pulse chronoamperometry were optimized towards the most-sensitive voltammetric detection of 4-nitrophenol (4-NP). The resulting electrodes were characterized by scanning electron microscope with energy dispersive X-ray spectroscopy. While 4-NP could not be detected by bare nonactivated vAuEs at all, their electrochemical activation offered limit of detection (LoD) 25 and $5 \mu \mathrm{mol}^{-1}$ by means of CV and DPV, respectively. Herein introduced AgAP electrodeposited on vAuE offered 2.5-times lower LoDs $10 \mu \mathrm{mol} .1^{-1}$ by CV and comparable LoD $5 \mu \mathrm{mol} .1^{-1}$ by DPV. Advantageously, AgAPs could be repeatedly deposited on and anodically dissolved from the vAuE with relative standard deviation $13 \%$ of ten-times repeated DPV signal of 4-NP $\left(100 \mu \mathrm{mol} .1^{-1}\right)$. In coparison to vAuE, the vAuE-AgAP offered about $400 \mathrm{mV}$ broader
\end{abstract}


potential window and it allowed detection of single strand DNA fragment labeled by osmium tetroxide-bipyridine complex down to $2 \mathrm{ng} \cdot \mu \mathrm{l}^{-1}$ by means of DPV.

Keywords: DNA, Gold, 4-nitrophenol, Silver amalgam, Voltammetry

\section{Highlights:}

- $\quad$ Thin gold film electrodes (vAuE) represent suitable substrate for electrodeposition of silver amalgam particles (AgAP)

- Metal composition and morphology of the electrode surface was observed by SEM-EDS

- $\quad$ vAuE-AgAP represents novel electrode material for electrochemically reducible organic nitro compounds

- $\quad$ AAuE-AgAP allowed detection of Os,bipy-labeled DNA by means of differential pulse voltammetry down to $2 \mathrm{ng} \cdot \mu \mathrm{l}^{-1}$

Dedicated in memory of prof. Emil Paleček.

\section{Introduction}

Recent progress in molecular diagnostics has led to discoveries of new genes, proteins, and other biomarkers, which were shown to be involved in various diseases, carrier detection, and in primary patient or prenatal diagnosis.[1-3] Electrochemical sensors and biosensors represent a perspective tool for this purpose.[4] In electroanalysis of deoxyribonucleic acid (DNA), ribonucleic acid (RNA) and protein or glycoprotein, dropping and hanging mercury drop electrodes still keep their irreplaceable importance.[2,3,5] High hydrogen overpotential, together with strong adsorption of the above-mentioned biopolymers on the ideally smooth and easily renewable electrode surface, [6] allow their highly sensitive detection based on intrinsic redox and capacitive tensammetric peaks or on so-called $\mathrm{H}$ peak, related to catalytic hydrogen evolution reaction (CHER). Adsorptive transfer stripping technique [7] combined with suitable voltammetric method or chronopotentiometric stripping analysis at HMDE might be used in detection or structural studies of both label-free or labeled biopolymers.[7,8] However, low mechanical stability, fear of mercury vapor toxicity, and legislative limitations hinder its practical applications and further development of DNA and protein sensors. Recent progress in this field combines research in chemistry, biochemistry, physical chemistry and material sciences, where nanotechnology and metal 
nanoparticles take advantages from their exceptional properties, e.g. high surface area, biocompatibility, catalytic effects, enhancement of charge transfer or unique optical properties. Metal nanoparticles can be used for: i) immobilization or labeling of biomolecules; ii) catalysis of electrochemical reactions; iii) enhancement of electron transfer; and iv) they can act as reactants/agents, e.g. antibacterial, antifungal, antiviral, anti-inflammatory, anti-angiogenic, and anti-cancer.[9-12] A very promising application of the nanotechnology consists in fabrication of micro- or even nano- electrode arrays in combination with high throughput microfluidic or "labon-chip" systems [13-15], which might be combined with electrochemical, optical (e.g. fluorescence, Raman, surface plasmon resonance (SPR) spectroscopies) or even spectroelectrochemical (UV/VIS, electrochemiluminiscence) detection. Attributes of metal mercury and silver nanoparticles might be advantageously combined to provide sufficient mechanical stability of the material and to enable novel possibilities for fabrication of nanostructured silver amalgam electrode arrays or other sensing architectures with promising electrochemical and spectroscopic properties.

Electroanalytical applications of silver amalgam electrodes prepared by mixing of the individual metals have been demonstrated,[16-19] but miniaturization of these electrodes is rather difficult. Bimetallic Ag-Hg core-shell nanoparticles can be prepared by chemical co-reduction of the metal ions $\left(\mathrm{Hg}^{2+}\right.$ and $\left.\mathrm{Ag}^{+}\right)$in one solution.[20,21] Metal displacement [22] represents a simple alternative to fabrication of voltammetric and/or amperometric detectors based on needle-like silver amalgam crystals, which have been applied in batch and/or flow-through HPLC analysis of environmental pollutants, 4-nitrophenol [23] and dinitronaphthalenes [24], respectively.

Amongst the above mention procedures, very promising results have been obtained by the electrochemical deposition of silver amalgam particles (AgAPs) onto various conductive supporting materials. Up to date, indium tin oxide (ITO) transparent electrode [25], glassy carbon (GC) [26] or pyrolytic graphite electrodes (PGE) [27] have already been used as substrates, and the respective conditions of the electrodeposition have been optimized. ITO and PGE electrodes decorated by AgAPs were found to be suitable in voltammetric and spectroelectrochemical detection of organic nitro compounds. The GC with electrodeposited AgAP was used for indirect determination of cysteine, glutathione, and metallothioneins by measuring the catalytic hydrogen evolution signal according to the Brdička reaction using cobalt as a catalyst.[26] Thanks to 
preferential adsorption of fluorescently labeled calf thymus DNA and green fluorescent protein (GFP) on AgAP electrodeposited on PGE, intrinsic DNA redox signals (cathodic peak of cytosine and adenine, and anodic peak of guanine's reduction products) and catalytic peak $\mathrm{H}$ offered by GFP could thus be observed.[25,27] Analogical CHER response of bovine serum albumin protein could be observed down to $5 \mathrm{nmol} \cdot \mathrm{l}^{-1}$ using ultrathin $(<1 \mu \mathrm{m})$ silver solid amalgam electrode arrays, prepared by vacuum silver metal sputtering onto a glass substrate, photolithography and galvanic mercury amalgam formation.[28] However, the electrodeposited metal mercury slowly soaked into the silver film, which negatively influenced its morphology and final composition of the electrode surface and thus hindered its broader applications.

This work is focused on optimization of the electrodeposition of AgAP onto a thin gold film, vapordeposited on silicon wafers (vAgE). Herein utilized double pulse potentiostatic chronoamperometry (DPCA) was optimized towards suitable electrochemical response for model organic nitro compound, 4-nitrophenol (4-NP). Its sodium salt is widely used as a drug, colorant or component of various agrochemicals worldwide (Atonic ${ }^{\circledR}, \mathrm{N}$-phenol MIX ${ }^{\circledR}$ etc.)[29], even though the 4-NP is registered by US Environmental Protection Agency on the List of Priority Pollutants.[30,31] Morphological changes of the vAuE upon deposition of AgAPs (vAuE-AgAP) during the optimization of DPCA were observed by scanning electron microscopy with energy dispersive X-ray spectroscopy (SEM-EDS). Applicability of the optimized system was verified by voltammetric detection of 4-NP using cyclic voltammetry (CV) and differential pulse voltammetry (DPV). In addition, DPV detection of single-stranded DNA fragment chemically labeled by osmium tetroxide, bipyridine complex (Os,bipy) was investigated. Corrosion of the optimized vAuE-AgAP in acetate buffer $\mathrm{pH} 5.0$ have also been observed.

\section{Experimental}

\subsection{Chemicals and reagents}

Solution of 0.01 mol. ${ }^{-1} \mathrm{AgNO}_{3}$ (p.a. $99.8 \%$, Safina) in 0.1 mol. ${ }^{-1} \mathrm{KNO}_{3}$ (p.a. >98 \%, Fluka) was used. Solution of 0.01 mol. $1^{-1} \mathrm{Hg}\left(\mathrm{NO}_{3}\right)_{2}$ in $0.1 \mathrm{~mol}^{-1} \mathrm{KNO}_{3}$ was prepared by dissolution of exact amount of metal mercury (99.999\%, Polarografie Praha) in quantitative volume of concentrated $\mathrm{HNO}_{3}$ (p.a. 65\%, Sigma-Aldrich) in a fume hood, then a/the given amount of $\mathrm{KNO}_{3}$ and deionized 
water was added to reach the required concentrations. 4-NP was from Sigma-Aldrich(USA). Synthetic oligonucleotides (ONs) were purchased from Generi Biotech (Czech Republic). Aliquots of dissolved $\mathrm{ONs}$ were stored at $-20^{\circ} \mathrm{C}$ in a freezer. All solutions were prepared using deionized water (Millipore, Milli-Q water system, USA). Other chemicals were of analytical grade.

\subsection{Preparation of vapor-deposited gold thin film electrode}

The vAuEs were fabricated by deposition of $3 \mathrm{~nm}$ of titanium adhesion layer and $150 \mathrm{~nm} \mathrm{of} \mathrm{Au}$ film onto bare silicon wafers using electron-beam evaporation system (Bestec, Germany).

\subsection{Electrodeposition of AgAPs}

Electrodeposition of the AgAPs or silver-inly particles (AgP) was carried out in homemade Teflon cell with the specific surface area defined by an O-ring (i.d. $5 \mathrm{~mm}$ ) on a vAuE using DPCA from $0.01 \mathrm{~mol} . \mathrm{l}^{-1} \mathrm{Ag}^{+} / \mathrm{Hg}^{2+}$ or $0.01 \mathrm{~mol} . \mathrm{l}^{-1} \mathrm{Ag}^{+}$in 0.1 mol. $1^{-1} \mathrm{KNO}_{3}$, respectively. Electrodeposition of on vAuE Optimization of the DPCA parameters is embodied in the Chap. 3.2 and 3.3. The vAuE was used as the working electrode without prior pretreatment, $\mathrm{Ag} / \mathrm{AgCl} / 3 \mathrm{M} \mathrm{KCl}$ as the reference electrode and a platinum wire as the counter electrode.

\subsection{Voltammetric measurements}

Electrochemical measurements were carried out by Autolab PGStat128N potentiostat operated by Nova software Ver. 1.11 (both Metrohm-Autolab). The three-electrode system, involving vAuE or vAuE-AgAP as working, $\mathrm{Ag} / \mathrm{AgCl} / 3 \mathrm{M} \mathrm{KCl}$ as reference, and platinum rod as auxiliary electrode, was always used. Voltammetry of 4-NP at bare vAuE was done after its activation by cycling

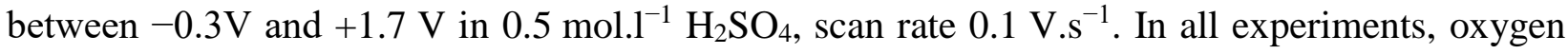
was removed from the respective solutions by passing a stream of argon saturated with water vapor through them for $5 \mathrm{~min}$. All experiments were performed at room temperature. CV measurements of 4-NP were performed with initial potential $0.0 \mathrm{~V}$, switching potential $-1.0 \mathrm{~V}$, scan rate $0.1 \mathrm{~V} . \mathrm{s}^{-1}$, background electrolyte $0.2 \mathrm{~mol}^{-1}$ acetate buffer $\mathrm{pH} 5.0$ (AcB). DPV measurements of 4-NP in $\mathrm{AcB}$ and of unlabeled and Os,bipy-labeled ON in background electrolyte Britton-Robinson (BR) buffer $\mathrm{pH} 4.0$ were performed with initial potential $0.15 \mathrm{~V}$, final potential $-1.85 \mathrm{~V}$, pulse amplitude $0.025 \mathrm{~V}$, scan rate $0.05 \mathrm{~V} . \mathrm{s}^{-1}$. Dissolution of the AgAP in repeatability experiments was carried out by potentiostatic chronoamperometry with applied potential $+0.5 \mathrm{~V}$ during $60 \mathrm{~s}$ in 0.1 mol. $1^{-1}$ $\mathrm{HNO}_{3}$. 


\subsection{SEM-EDS analysis}

The morphology of the samples was investigated by SEM (FEI Verios 460L) equipped with an EDS system (EDAX SDD Octane Super). The acceleration voltage of $6.0 \mathrm{kV}$ and beam current of $0.80 \mathrm{nA}$ were applied. $\mathrm{Ag} \mathrm{L} \alpha, \mathrm{Hg} \mathrm{M} \alpha$ and $\mathrm{Au} \mathrm{M} \alpha$ spectral lines were used for the EDS quantification using peak-to-background PeBaZAF method with a correction for rough surfaces/particles.

\subsection{Modification of Os, bipy}

Os,bipy was prepared by mixing of equimolar concentration of osmium tetroxide with 2,2'bipyridin (Sigma-Aldrich). Synthetic oligonucleotide 4basII (5'CTAGCATGAGCTCAGTCCCATGCCGCCCATG-3') and homo-oligonucleotide A30 were incubated with $2 \mathrm{mmol} . \mathrm{I}^{-1}$ Os,bipy in $0.1 \mathrm{~mol} . \mathrm{I}^{-1}$ Tris buffer $\mathrm{pH} 7.3$ for $30 \mathrm{~min}$ at $37^{\circ} \mathrm{C}$. Unreacted Os,bipy was removed from the solution by dialysis against 0.1 mol. $1^{-1}$ Tris $\mathrm{pH} 7.3$ for 16 hours.

\section{Results and Discussion}

\subsection{Electrochemical behavior of $\mathrm{Ag}^{+}$and $\mathrm{Hg}^{2+}$ at $\mathrm{vAuE}$}

In order to observe the electrochemical behavior of the individual $\mathrm{Ag}^{+}$and $\mathrm{Hg}^{2+}$ ions at bare vAuE in 0.1 mol..$^{-1} \mathrm{KNO}_{3}$, corresponding cyclic voltammograms were registered. A mixture of both metals $\left(\mathrm{Ag}^{+} / \mathrm{Hg}^{2+}, 30.6 \mathrm{w} \% \mathrm{Ag}\right)$ was also registered within potential ranges of $+1.5 \mathrm{~V}$ to $-1.5 \mathrm{~V}$ (Fig. 1). According to this $\mathrm{CV}$, intervals of nucleation $\left(E_{l}=\{0.2 ;-0.6\} \mathrm{V}\right)$ and grow potentials $\left(E_{2}=\{-1.0 . ;-2.0\} \mathrm{V}\right)$ of DPCE could be selected in consideration of the negative end of the potential window limited by $\mathrm{H}^{+}$reduction, and of the $\mathrm{Ag}^{+}$and $\mathrm{Hg}^{2+}$ reduction at bare $\mathrm{vAuE}$, respectively. 


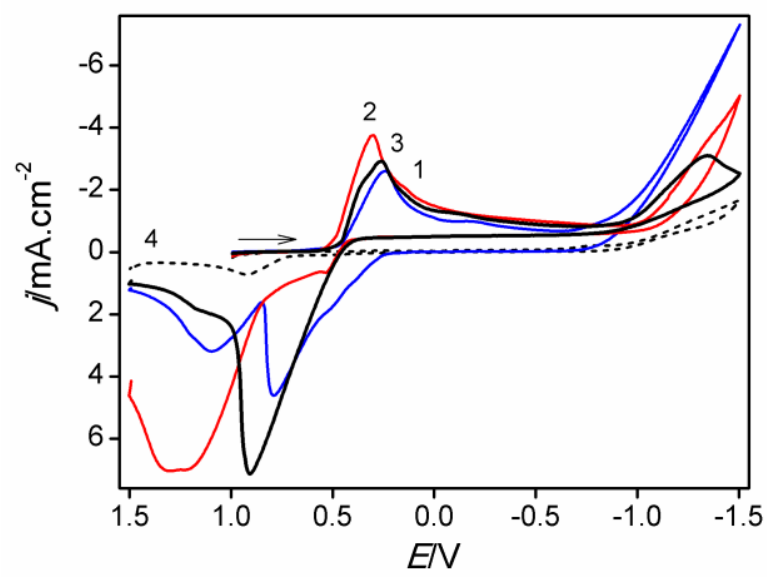

Fig. 1. A) Cyclic voltammograms of: $0.01 \mathrm{~mol} . \mathrm{l}^{-1} \mathrm{AgNO}_{3}$ (1), $0.01 \mathrm{~mol} . \mathrm{l}^{-1} \mathrm{Hg}\left(\mathrm{NO}_{3}\right)_{2}$ (2), and $0.01 \mathrm{~mol}^{-1} \mathrm{AgNO}_{3}+\mathrm{Hg}\left(\mathrm{NO}_{3}\right)_{2} 30.6 \mathrm{w} \% \mathrm{Ag}$ (3) in $0.1 \mathrm{~mol}^{-1} \mathrm{KNO}_{3}$ (4). Initial potential $1.0 \mathrm{~V}$, switching potential $-1.5 \mathrm{~V}$, final potential $1.5 \mathrm{~V}$, scan rate $0.1 \mathrm{~V} . \mathrm{s}^{-1}$.

Overlapping cathodic peaks of $\mathrm{Ag}^{+}$and $\mathrm{Hg}^{2+}$ are in good agreement with the close values of their standard redox potentials $\left\{\mathrm{E}^{0}\left(\mathrm{Ag}^{+} / \mathrm{Ag}\right)=0.7991 \mathrm{~V}, \mathrm{E}^{0}\left(\mathrm{Hg}^{2+} / \mathrm{Hg}\right)=0.7880 \mathrm{~V}\right.$; at $25^{\circ} \mathrm{C}$, vs. $\mathrm{SHE}$ [32]\}. Anodic dissolution of the electrodeposited metal mercury at vAuE is significantly shifted towards more positive potentials, in comparison to anodic dissolution of Ag, due to formation of $\mathrm{Au}-\mathrm{Hg}$ amalgam. Unfortunately, only one $\mathrm{CV}$ of $\mathrm{Hg}^{2+} / \mathrm{Hg}^{0}$ could thus be observed at vAuE before a complete disruption of the gold film caused by its intensive amalgamation. In case, when only a/the much thinner mercury film is electrodeposited on the thicker Ag or Au film electrodes, the mercury slowly soaks into the underlying metal and results in unwanted time-depending changes of the amalgam morphology associated with the amalgam composition on the surface. Therefore, electrochemical preparation of the silver or gold amalgam by electrochemical reduction of the only $\mathrm{Hg}^{2+}$ at $\mathrm{Ag}$ or $\mathrm{Au}$ thin film electrodes is not convenient. Advantageoulsy, the electrochemical codeposition of metal mercury and silver provides the most preferential/stable forms of amalgam, which are chemically and mechanically stable and disruption of the vAuE is negligible. This procedure brings perspective opportunity in preparation of silver amalgam on the top of the gold film. Solution composition $\left(\mathrm{Ag}^{+} / \mathrm{Hg}^{2+}\right.$ ratio) and individual parameters of the DPCA have to be optimized and resulting surfaces characterized for their further application. 


\subsection{Influence of solution constitution}

In analogy with the application of DPCA in preparation of AgAPs on ITO [25], the same method was applied for electrodeposition of AgAPs on vAuEs in this work. Nucleation potential $\left(E_{1}\right)$ $-1.5 \mathrm{~V}$ applied for nucleation time $\left(t_{1}\right)\left(t_{l}=50 \mathrm{~ms}\right.$ was kept constant during all the experiments), followed by growth potential $\left(E_{2}\right) 0.0 \mathrm{~V}$ lasting growth time $\left(t_{2}\right) 60 \mathrm{~s}$, were selected at the beginning to observe the influence of the solution constitution on the resulted composition of AgAPs. Content of the $\mathrm{Ag}^{+}$in the solution varied between $8.7-61.7 \mathrm{w} \% \mathrm{Ag}$, whereas the mercury ions constituted the rest and net content of both metals was kept constant at 0.01 mol. $1^{-1}$. SEM micrographs of the resulting electrodes (Fig. 2) reveal the significant difference in the surface morphology of the electrodes based on the solution composition. The desired small and homogenously distributed AgAPs were observed for the deposition solutions with silver concentration in the range of $20-$ $40 \mathrm{w} \% \mathrm{Ag}$. The most dense and homogenous surface with the smallest AgAPs was obtained for the solution containing $30.6 \mathrm{w} \% \mathrm{Ag}$ (Fig. 2d). Resulting dense and size of the AgAPs influence electrochemical properties of the system (supporting material vs. AgAPs). When supporting material offers signal of the analyte at notably more negative potentials and surface coverage of the AgAPs is not sufficient, two signals of the analyte appear (one on AgAPs and one on the supporting material), what deteriorate and electroanalytical performance of the method. Moreover, increasing dense and decreasing size of the AgAPs is in conformity with requirements for potential application in optical and spectro-electrochemical systems (e.g. a dark-field spectroscopy shows surface plasmon resonances of AgAPs on ITO in visible range of wavelengths for particle size below $200 \mathrm{~nm}$ ).[33] According to these requirements and to the highest and the most welldeveloped CV signals of 4-NP, the deposition solution containing $30.6 \mathrm{w} \% \mathrm{Ag}$ was selected for further optimization of DPCA parameters in following Chapter 3.3.

The EDS analysis of the AgAP brought interesting information. The ultimate mercury content in the AgAPs varied between 30 to $40 \mathrm{w} \% \mathrm{Hg}$ within the whole studied $\mathrm{Ag}^{+}$content $(8.7-61.7 \mathrm{w} \% \mathrm{Ag})$ in the deposition solution, what signifies formation of the AgAPs in preferential composition. Silver varied between 10 to $40 \mathrm{w} \% \mathrm{Hg}$ with the maximum at $22.5 \mathrm{w} \% \mathrm{Ag}$ in the solution, while $\mathrm{Au}$ varied within the range $60-30 \mathrm{w} \% \mathrm{Au}$ with the minimum at $22.5 \mathrm{w} \% \mathrm{Ag}$. High content of $\mathrm{Hg}^{2+}$ in the solution caused more significant dissolution of the Au film and thus higher content of the Au in the AgAPs. Moreover, the gold contamination of the AgAPs was caused by small mercury 
droplets, which are formed as the first ones during the nucleation pulse and thus can easily dissolve the gold film.[33,34] This contamination decreased during the prolonged growth pulse (Fig. S3), when $\mathrm{Ag}^{+}$and $\mathrm{Hg}^{2+}$ are predominantly reduced.
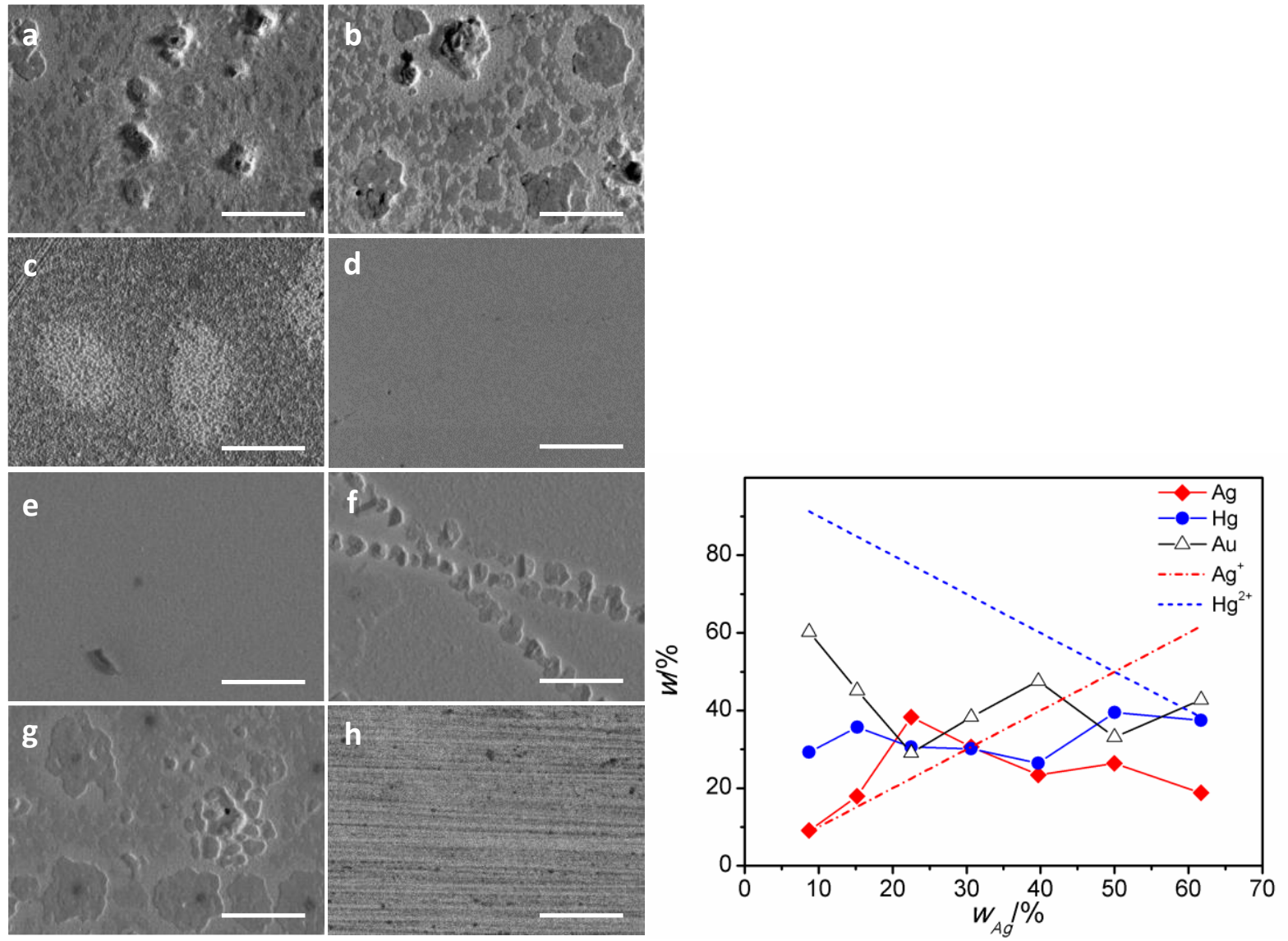

Fig. 2. SEM micrographs of AgAPs electrodeposited onto vAuE from solution containing ( $w_{\mathrm{Ag}}$ in \%): 8.7 (a), 15.2 (b), 22.5 (c), 30.6 (d), 39.7 (e), 50.0 (f), 61.7 w\%Ag (g) and bare vAuE (h), using $\operatorname{DPCA}\left(E_{1}=-1.5 \mathrm{~V}, t_{1}=50 \mathrm{~ms}, E_{2}=0.0 \mathrm{~V}\right.$ and $\left.t_{2}=60 \mathrm{~s}\right)$, scale bar $5 \mu \mathrm{m}$ and corresponding influence of electrodeposited amalgam's composition $(w)$ on solution constitution ( $w_{A g}$ ) resulting from EDS analysis. Straight lines indicate the content of the metals in the growth solution.

Influence of the deposition solution composition $\left(w_{A g}\right)$ on the electrochemical behavior of 4-NP was studied by CV on vAuE-AgAPs and compared to a bare activated vAuE (Fig. 3A). Note, that nonactivated bare vAuE did not give any electrochemical response to 4-NP in AcB. A cathodic peak of 4-NP appeared at potential $-650 \pm 35 \mathrm{mV}$ on vAuE-AgAPs, which is about $70 \mathrm{mV}$ more 
negative than in the case of a bare activated vAuE. Peak current of 4-NP did not significantly change within the studied range of Ag content in deposition solution and it was comparable with the peak current obtained on bare activated vAuE (Fig. 3B). This obviously corresponds to negligible changes of electro-active surface areas caused by the AgAPs.
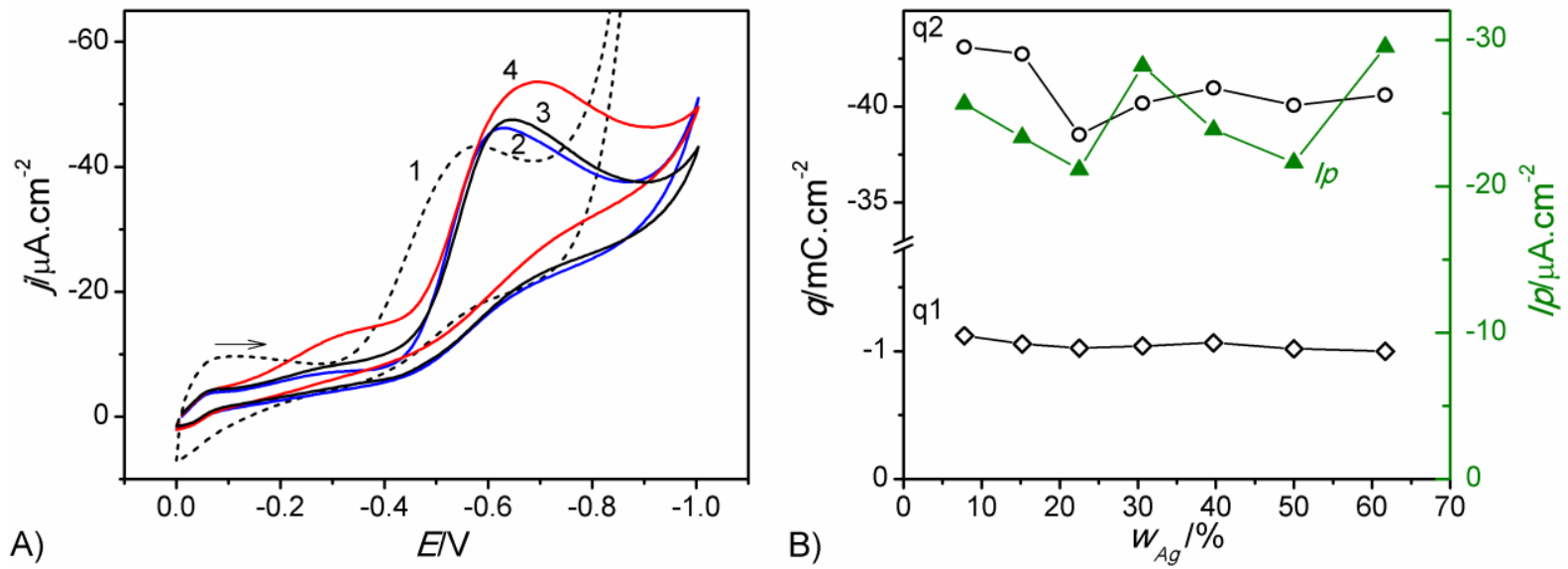

Fig. 3. (A) Cyclic voltammograms of $100 \mathrm{~mol}^{-1} \mathrm{l}^{-1} \mathrm{-NP}$ in $\mathrm{AcB}$ (scan rate $0.1 \mathrm{~V} \mathrm{~s}^{-1}$ ) registered by: $\mathrm{vAuE}(1)$ and at vAuE-AgAP prepared by DPCA using $E_{1}=-1.5 \mathrm{~V}, t_{1}=50 \mathrm{~ms}, E_{2}=0.0 \mathrm{~V}$ and $t_{2}=60 \mathrm{~s}$, from the solution containing: 15.2 (2), 30.6 (3) and $50.0 \mathrm{w} \% \mathrm{Ag}$ (4) in $0.1 \mathrm{~mol}^{-1} \mathrm{KNO}_{3}$. (B) Extracted charge densities corresponding to the initial $(q 1)$ and grow $(q 2)$ DPCA pulses consumed during the electrodeposition of AgAP in dependence on the solution constitution $\left(w_{A g}\right)$ and correlated to 4-NP peak currents (Ip) evaluated from the CVs (A) registered on vAuE (a, dash line) and vAuE-AgAP (b).

\subsection{Optimization of electrodeposition parameters}

The DPCA parameters: $E_{1}, E_{2}, t_{2}$ were optimized using the deposition solution containing $30.6 \mathrm{w} \%$ Ag. Theoretically, the nucleation potential influences the number of nucleation centers (and therefore also the final particle density) and the grow potential influences the speed of the particle growth and also their composition.[35] SEM images of the electrodeposited AgAPs obtained during optimization of DPCA parameters at vAuE together with the corresponding charge densities ( $q 1$ and $q 2)$ and resulting metal content ( $\mathrm{Ag}, \mathrm{Au}, \mathrm{Hg}$ ) are shown on Fig. S1-S3. Effects of these parameters on their electrochemical behavior were studied by CV of 4-NP in AcB (Fig. 4). 


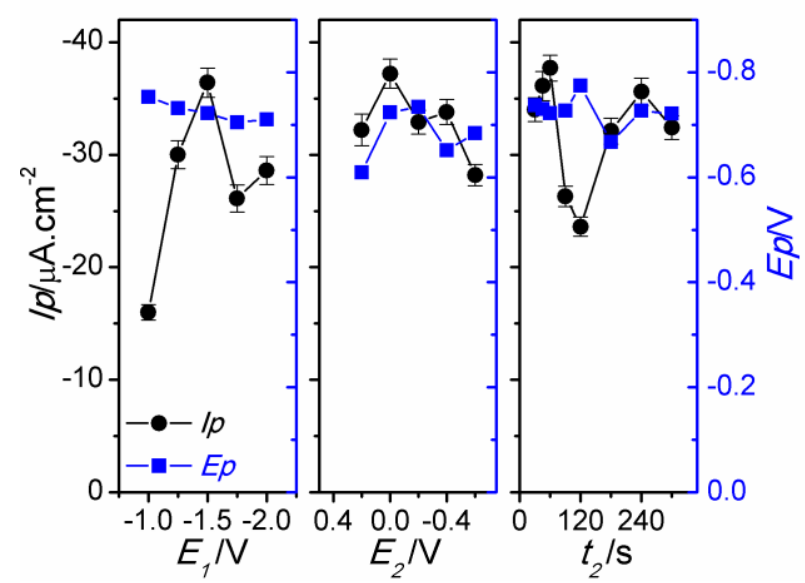

Fig. 4. Effect of nucleation potential $\left(E_{1}\right)$, grow potential $\left(E_{2}\right)$ and grow time $\left(t_{2}\right)$ on peak current (Ip) and potential (EP) of $100 \mu \mathrm{mol} .1^{-1}$ 4-NP registered by CV at vAuE-AgAP in AcB. The vAuEAgAPs were prepared by DPCA with variable $E_{1}$ (constant $t_{1}=50 \mathrm{~ms}, E_{2}=0.0 \mathrm{~V}$ and $t_{2}=60 \mathrm{~s}$ ), $E_{2}$ (constant $E_{1}=-1.5 \mathrm{~V}, t_{1}=50 \mathrm{~ms}$ and $t_{2}=60 \mathrm{~s}$ ) and $t_{2}\left(\right.$ constant $E_{l}=-1.5 \mathrm{~V}, t_{1}=50 \mathrm{~ms}$ and $E_{2}=0.0 \mathrm{~V}$ ) from $0.01 \mathrm{~mol} .1^{-1} \mathrm{Ag}^{+} / \mathrm{Hg}^{2+}(30.6 \mathrm{w} \% \mathrm{Ag})$ in $0.1 \mathrm{~mol} .1^{-1} \mathrm{KNO}_{3}$.

The highest and most pronounced CV peak of 4-NP was observed at vAuE-AgAP with high density of the smallest particles, obtained by DPCA with the following parameters: $E_{1}=-1.5 \mathrm{~V}, t_{1}=50 \mathrm{~ms}$, $E_{2}=0.0 \mathrm{~V}$ and $t_{1}=60 \mathrm{~s}$. These parameters were therefore selected for the subsequent measurements of 4-NP concentration dependences using CV and DPV and also for the further corrosion studies. Moreover, some of the further experiments were compared with the AgP electrodeposited on vAuE (vAuE-AgP) using the same DPCA parameters.

\subsection{Voltammetric detection of 4-nitrophenol}

Utilization of the vAuE-AgAPs in flow-through or microfluidic detection systems could be one of the perspective applications of the vAuE-AgAP in the future. Therefore, we investigated the repeatability of the AgAP electrodeposition/4-NP detection and AgAP electro-dissolution process on the vAuE. Ten-fold deposition of AgAPs, DPV detection of 4-NP, and anodic dissolution of the AgAP was repeated ten times. The resulting relative standard deviation (RSD) of the 4-NP's DPV signal was $13 \%$ (Fig. 5A). RSD values of the corresponding charge densities consumed by the AgAP deposition were les then $1.2 \%$ for nucleation pulse and $2.2 \%$ for the grow pulse, which is a 
warning sign indicating imperfect deposition of AgAPs. Even though the SEM images revealed some surface defects on the surface of gold film after the experiments, the observed repeatability of the 4-NP detection by DPV at vAuE-AgAP provides perspective application in development of flow-through systems with repeatedly renewed AgAP inside of the electrochemical cell. This will be the scope of further research.

Applicability of optimized vAuE-AgAP in electroanalysis, concentration dependences of the 4-NP were registered by $\mathrm{CV}$ and $\mathrm{DPV}$ at bare vAuE, bare activated vAuE and vAuE-AgAP in AcB (Fig. 5B). The whole concentration dependences were measured on the vAuE with particles electrodeposited only once. Washing by deionized water was found to be sufficient for the desired three-fold repeatability of the measurements with RSD not exceeding 5\% for both low $\left(10 \mu \mathrm{mol} .1^{-1}\right)$ or high $\left(100 \mu \mathrm{mol} . \mathrm{I}^{-1}\right)$ concentration of 4-NP. Parameters of the linear fit of the concentration lines, together with the resulting limits of detection (LoD, calculated as LoD $=3 \times$ Signal/Noise ${ }^{[36]}$, are summarized in Tab. I.
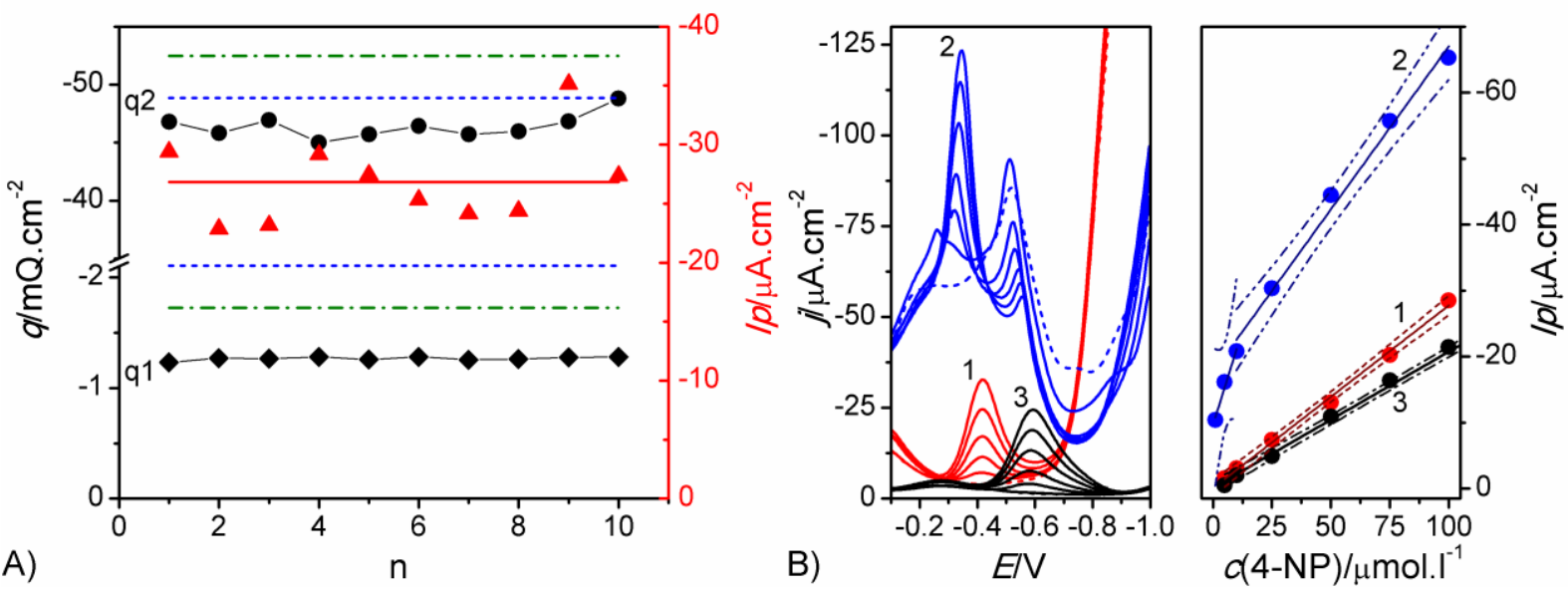

Fig 5. A) Repeatability of a ten-times repeated AgAP electrodeposition on the same vAuE with electrochemical dissolution of the AgAP between each measurement, showing ten consecutive charge densities ( $q 1$ and $q 2$ ) with RSD 1.1 and 2.2\% and regulation diagram of ten time repeated DPV detection of 4-NP $\left(100 \mu \mathrm{mol} . \mathrm{l}^{-1}\right)$ representing evaluated peak current (Ip) and average (solid line) together with warning (dash lines, $\pm 2 \times \mathrm{SD}$ ) and regulation limits (das dot line, $\pm 3 \times \mathrm{SD}$ ). B) Differential pulse voltammograms of 4-NP in dependence on its concentration $(0 ; 1 ; 10 ; 25 ; 50 ; 75$ and $100 \mu \mathrm{mol.} \mathrm{I}^{-1}$ ) measured at: vAuE (1), vAuE-AgP (2) and vAuE-AgAP (3) (DPCA: $E_{1}-1.5 \mathrm{~V}$,

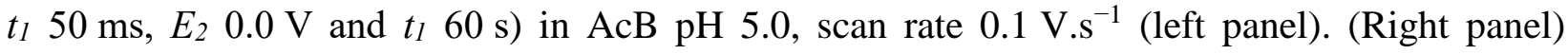


Extracted concentration dependences of 4-NP with concentration straight lines (solid lines) and 95$\%$ confidence bands (dash-dot lines).

Tab. I Parameters of the linear fit of concentration lines for detection of 4-NP using CV or DPV at selected electrodes, all measured in 0.2 mol.1 ${ }^{-1} \mathrm{AcB} \mathrm{pH}$ 5.0.

\begin{tabular}{|c|c|c|c|c|c|c|}
\hline Method & Electrode & $\begin{array}{l}\text { Slope/ } \\
\mu \mathrm{A} \cdot 1 . \mu \mathrm{mol}^{-1} \cdot \mathrm{cm}^{-2}\end{array}$ & $\begin{array}{l}\text { Intercept/ } \\
\mu \mathrm{A} \cdot \mathrm{cm}^{-2}\end{array}$ & $\mathrm{R}^{2}$ & $\begin{array}{l}\text { Range/ } \\
\mu \mathrm{mol}^{-1} \mathrm{I}^{-1}\end{array}$ & $\begin{array}{l}\text { LoD/ } \\
\mu \mathrm{mol}^{-1} 1^{-1}\end{array}$ \\
\hline \multirow[t]{3}{*}{$\mathrm{CV}$} & $\mathrm{vAuE}$ & $-0.382 \pm 0.012$ & $0^{\mathrm{b}}$ & 0.9900 & $25-200$ & 25 \\
\hline & vAuE-AgP & $-0.577 \pm 0.019$ & $-1.22 \pm 0.97$ & 0.9938 & $5-100$ & 5 \\
\hline & vAuE-AgAP & $-0.592 \pm 0.0085$ & $0^{b}$ & 0.9990 & $5-200$ & 10 \\
\hline \multirow[t]{4}{*}{ DPV } & $\mathrm{vAuE} \mathrm{E}^{\mathrm{a}}$ & $-0.2779 \pm 0.0079$ & $0^{b}$ & 0.9957 & $5-100$ & 5 \\
\hline & vAuE-AgP & $-1.14 \pm 0.14$ & $9.67 \pm 0.93$ & 0.9691 & $1-10$ & 1 \\
\hline & vAuE-AgP & $-0.494 \pm 0.027$ & $17.6 \pm 1.7$ & 0.9878 & $10-100$ & - \\
\hline & vAuE-AgAP & $-0.206 \pm 0.0042$ & $0^{\mathrm{b}}$ & 0.9974 & $1-200$ & 5 \\
\hline
\end{tabular}

Our vAuE-AgAPs provided the broadest linear concentration range from 5 to $200 \mu \mathrm{mol} .1^{-1}$ of 4$\mathrm{NP}$ by means of $\mathrm{CV}$ with the LoD of $10 \mu \mathrm{mol} . \mathrm{l}^{-1}$. Its sensitivity was almost twice as high as the bare activated vAuE with its LoD being only $25 \mu \mathrm{mol} .1^{-1}$. On the other hand, it was worse than the sensitivity of vAuE-AgP, which offered twice as low LoD of $5 \mu \mathrm{mol} .1^{-1}$. DPV method has also been utilized for detection of 4-NP at all three electrodes mentioned above. The vAuE-AgAP provided the broadest linear concentration range $1-200 \mu \mathrm{mol} .1^{-1}$ with the LoD of $5 \mu \mathrm{mol} .1^{-1}$. The same LoD of $5 \mu \mathrm{mol} .1^{-1}$ was achieved at bare activated vAuE, but only within the linear concentration range $1-100 \mu \mathrm{mol} .1^{-1}$. The vAuE-AgP offered the lowest LoD of $1 \mu \mathrm{mol} .1^{-1}$, but only within the narrow linear concentration range $1-10 \mu \mathrm{mol}^{-1}$. DP voltammograms of AcB showed some parasitic signal around $-0.5 \mathrm{~V}$, which height decreased with increasing 4-NP concentration. Origin of the peak is unknown, but it does not interfere with the peak of 4-NP. 
Obtained results do not reach the LoD achieved by some more sensitive detection systems for 4NP [37], but prove the applicability of vAuE-AgAPs in organic electroanalysis of nitro compounds with LoDs down to micromolar concentrations and confirmed sufficient reproducibility of repeated AgAP deposition.

\subsection{Corrosion of AgAPs}

Linear sweep voltammetry allows us to study corrosion of AgAPs in AcB and compare it with pure silver particles (AgPs) electrodeposited on vAuE by means of the same procedure (Fig. 6). From the measured data, summarized in Table II, several comments about corrosion of these nanomaterials can be mad

e: i) AgAPs are by about $130 \mathrm{mV}$ more stable towards their oxidation by air oxygen in the given solution then AgPs (compare their corrosion potentials, Ecorr). This indicates stabilization of AgPs by the process of amalgamation, but it leads to significant increase of corrosion current (Jcorr), which increased about 175-times, while the corrosion rate increased about 50-times. Polarization resistance was found to be comparable for both surfaces, reaching values around $20 \mathrm{k} \Omega$.

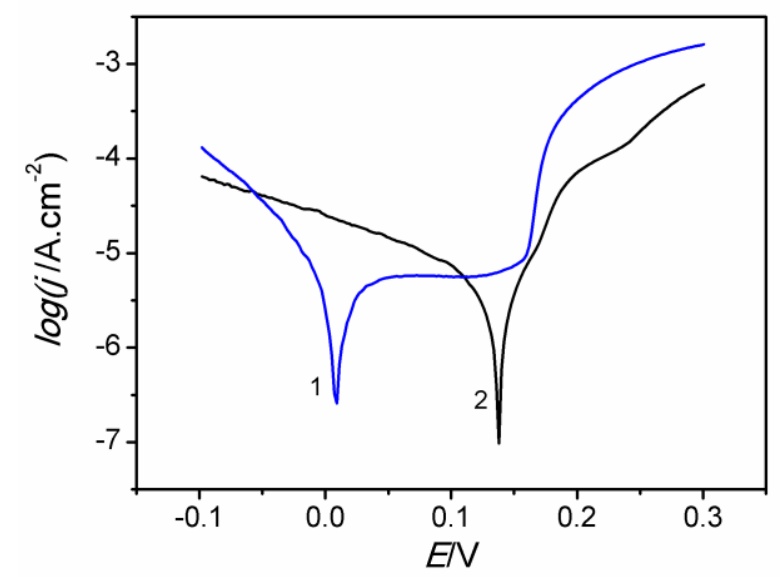

Fig. 6 Tafel plots representing corrosion of AgP (1) and AgAP (2) electrodeposited on vAuE in $\mathrm{AcB}$, scan rate $0.1 \mathrm{~V} . \mathrm{s}^{-1}$ (parameters of DPCA: $E_{l}=-1.5 \mathrm{~V}, t_{1}=50 \mathrm{~ms}, E_{2}=0.0 \mathrm{~V}$ and $t_{2}=60 \mathrm{~s}$ from $0.01 \mathrm{~mol}^{-1} \mathrm{AgNO}_{3}$ (1) and from $0.01 \mathrm{~mol}^{-1} \mathrm{AgNO}_{3}+\mathrm{Hg}\left(\mathrm{NO}_{3}\right)_{2} 30.6 \mathrm{w} \% \mathrm{Ag}$ (2), both in $\left.0.1 \mathrm{~mol}^{-1} \mathrm{KNO}_{3}\right)$. 
Tab. II Corrosion parameters of $\mathrm{AgP}$ and $\mathrm{AgAP}$ at $\mathrm{vAuE}$ in $\mathrm{AcB}$ extracted from the Tafel plots (Fig. 6).

\begin{tabular}{|c|c|c|}
\hline Parameter & vAuE-AgP & vAuE-AgAP \\
\hline$\beta \mathrm{a} / \mathrm{mV} \cdot \mathrm{dec}^{-1}$ & $140 \pm 11$ & $237 \pm 19$ \\
\hline$\beta \mathrm{c} / \mathrm{mV} \cdot \mathrm{dec}^{-1}$ & $-178 \pm 13$ & $79.1 \pm 6.7$ \\
\hline Ecorr $/ \mathrm{mV}$ & $8.30 \pm 0.34$ & $137.7 \pm 8.9$ \\
\hline Jcorr $/ \mathrm{nA} \cdot \mathrm{cm}^{-2}$ & $7.34 \pm 0.31$ & $1289 \pm 53$ \\
\hline Corrosion rate $/ \mu \mathrm{m}$.year ${ }^{-1}$ & $0.304 \pm 0.021$ & $15.12 \pm 0.96$ \\
\hline Polarization resistance $/ \mathrm{k} \Omega$ & $19.8 \pm 1.3$ & $20.0 \pm 1.1$ \\
\hline
\end{tabular}

\subsection{Voltammetric detection of Os,bipy-labeled oligonucleotides}

Direct detection of the ONs by $\mathrm{CV}$ at vAuE-AgAP using their intrinsic redox-active signals related to presence of nucleobases: cytosine, adenine and guanine [2] was not successful. Thanks to specific reactivity of pyrimidine nucleobases towards Os, bipy, ONs might be chemically modified by this redox-active tag. The $\mathrm{C} 5=\mathrm{C} 6$ double bond in the pyrimidine ring of thymin is the most readily modified one by Os, bipy, followed by uracil and 5-methyl cytosine, whereas cytosine reacts rather slowly. [38,39] This approach was used for detection of specific oligodeoxynucleotide sequences. In our case, an oligonucleotide 4basII (5'CTAGCATGAGCTCAGTCCCATGCCGCCCATG-3'), which contains six thymine sites for Os,bipy modification, was modified with Os,bipy . Homo-oligonucleotide A30, which does not react with Os,bipy, was selected as the negative control. Fig. 7A shows that 4basII-Os, bipy yields two well-developed DPV peaks at $\mathrm{pH} 4$ : peak I at $-0.1 \mathrm{~V}$ and peak II at $-0.55 \mathrm{~V}$. The A30 treated under the same conditions with Os,bipy produced no peaks, same as the unmodified 4 basII. Voltammograms of unmodified 4basII and 4basII-Os, bipy show considerable differences between potentials corresponding to the start of CHER. In the presence of Os,bipy, hydrogen evolution started approximately at about $300 \mathrm{mV}$ less negative potentials due to the catalytic effect of Os. The voltammogram of 4 basII-Os,bipy measured on vAuE shows only peak I, peak II is not well visible due to the former catalytic hydrogen evolution on the Au substrate. These results establish vAuE-AgAP as a more responsive electrode for detection of Os, bipy-modified DNA compared to vAuE. A detailed view of the measured peaks II as a function of 4basII-Os,bipy concentration is 
shown on Fig. 7B. These experiment proved that vAuE-AgAP can be useful for DPV-based detection of 4 basII-Os, bipy down to $2 \mathrm{ng} \cdot \mu^{-1}$.
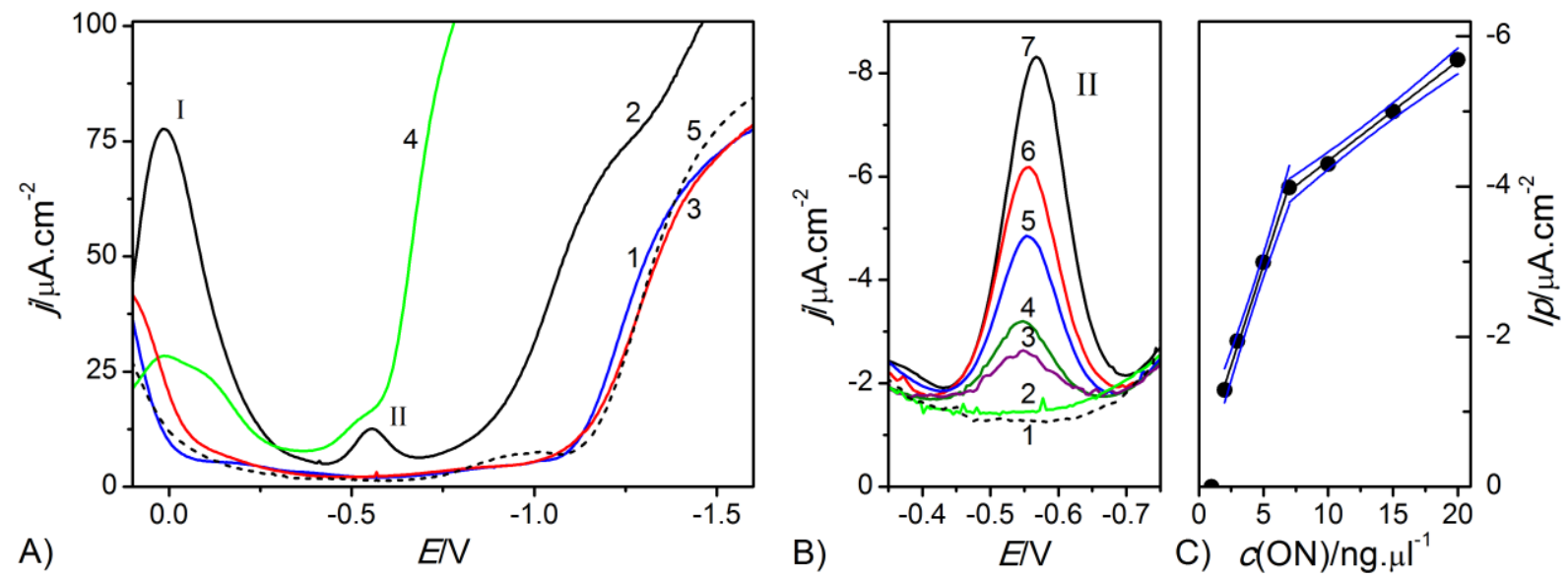

Fig. 7 (A) DP voltammograms of unmodified 4basII (1), Os, bipy modified 4basII (2) and Os, bipy modified A30 (3) at vAuE-AgAPs (prepared using DPCA, $E_{1}=-1.5 \mathrm{~V}, t_{1}=50 \mathrm{~ms}, E_{2}=0.0 \mathrm{~V}$, $t_{2}=60 \mathrm{~s}$ from $0.01 \mathrm{~mol} .1^{-1} \mathrm{Ag}^{+} / \mathrm{Hg}^{2+}(30.6 \mathrm{w} \% \mathrm{Ag})$ in $\left.0.1 \mathrm{~mol} .1^{-1} \mathrm{KNO}_{3}\right)$ and DPV of Os, bipy modified 4basII at vAuE (4) in BR buffer $\mathrm{pH} 4.0$ (5), concentration of all ONs was $20 \mathrm{ng} . \mu \mathrm{l}^{-1}$. (B) DP voltammograms of 4basII-Os,bipy with various concentration (ng. $\left.\mu \mathrm{l}^{-1}\right)$ : 0 (1), 1 (2), 2 (3), 3 (4), 5 (5), 10 (6) and 20 (7) at vAuE-AgAP in BR buffer pH 4.0. C) Extracted peak II current (Ip) of 4 basII-Os,bipy as a function of ON concentration.

Parameters of the linear fit of concentration lines for Os, bipy modified 4basII (ON) are as follows: $I p=0.1049 \times c(\mathrm{ON})+0.054 \mu \mathrm{A} \cdot \mathrm{cm}^{-2}\left(\mathrm{R}^{2}=0.9967\right.$, within $c(\mathrm{ON})$ from 3 to $\left.7 \mathrm{ng} \cdot \mu \mathrm{l}^{-1}\right)$ and $I p=0.0261 \times c(\mathrm{ON})+0.592 \mu \mathrm{A} \cdot \mathrm{cm}^{-2}\left(\mathrm{R}^{2}=0.9967\right.$, within $c(\mathrm{ON})$ from 7 to $\left.20 \mathrm{ng} \cdot \mu \mathrm{l}^{-1}\right)$. LoD of the Os,bipy modified 4basII was found to be $2 \mathrm{ng} \cdot \mu^{-1}$. Since Os, bipy binds to thymine in DNA and also to tryptophan molecules in peptides or proteins, it can serve as an electroactive chemical probe of the DNA or protein structures as well as their electroactive label $[40,41]$ or in the development of DNA hybridization sensors. [42,43] Utilization of the vAuE-AgAP in these applications will be the scope of further studies. 


\section{Conclusions}

In this paper, we investigated a possibility to electrodeposit mercury-based AgAPs on the $150 \mathrm{~nm}$ thin gold film as another then ITO and PGE supporting material what broaden its application possibilities in electroanalysis. Repeatable preparation and electrochemical dissolution of the AgAPs with the reproducibility up to $15 \%$ without the disruption of underlying vAuE was proved. Herein optimized vAuE-AgAP allowed application of CV and DPV in detection of model organic nitro compound at micromolar concentration level. The AgAPs are dissolved at potential about $150 \mathrm{mV}$ more positive than $\mathrm{AgPs}$ in $\mathrm{AcB}$, what reduce a speed of their spontaneous oxidation on the air. This resulted in an elimination of the anodic potential window offered by bare vAuE, but its applicable cathodic part of the potential window was broadened about $400 \mathrm{mV}$ towards negative potentials. This allowed sensitive detection of Os,bipy labeled ONs and broaden its application possibilities from organic nitro compounds to suitably labeled biopolymers.

In comparison to ITO, which is reduced and thus destroyed at potential more negative then $-1 \mathrm{~V}$ in aqueous media, the vAuE offer much broader negative potential window. It is comparable with bPGE, but bPGE is not suitable for automatized processes and preparation of thin films or patterns. Perspective advantage of the gold thin film substrate is based on its simple miniaturization and variability of electrode architecture design, which may be manufactured by photo lithography. This procedure could be advantageously utilized in development of optical, spectroelectrochemical or microfluidic devices applicable in further bioelectrochemical research of biopolymers (DNA, RNA, proteins, glycoproteins etc.). These topics are in the scope of our further reseach.

\section{Acknowledgment}

This work has been supported by a Grant Agency of the Czech Republic (project 17-23634Y). A part of this work was carried out with the support of CEITEC Nano Research Infrastructure (ID LM2015041, MEYS CR, 2016-2019), CEITEC Brno University of Technology.

\section{Supporting information}


Fig. S1 SEM images of vAuE-AgAP registered during optimization of nucleation potential $\left(E_{1}\right)$ and registered charge density ( $q 1$ and $q 2)$ and composition $(w)$ of electrodeposited amalgams during the optimization of $E_{l}$.

Fig. S2 SEM images of vAuE-AgAP registered during optimization of grow potential $\left(E_{2}\right)$ and registered charge density ( $q 1$ and $q 2)$ and composition $(w)$ of electrodeposited amalgams during the optimization of $E_{2}$.

Fig. S3 SEM images of vAuE-AgAP registered during optimization of grow time $\left(t_{2}\right)$ and registered charge density ( $q 1$ and $q 2)$ and composition $(w)$ of electrodeposited amalgams during the optimization of $t_{2}$.

\section{References}

[1] F. Lucarelli, G. Marrazza, A. P. F. Turner, M. Mascini, Biosens. Bioelectron. 2004, 19, $515-530$.

[2] E. Palecek, M. Bartosik, Chem. Rev. 2012, 112, 3427-3481.

[3] E. Palecek, J. Tkac, M. Bartosik, T. Bertok, V. Ostatna, J. Palecek, Chem. Rev. 2015, 115, 2045-2108.

[4] M. Freitas, H. P. A. Nouws, C. Delerue-Matos, Electroanalysis 2018, 30, 1576-1595.

[5] E. Palecek, F. Scheller, J. Wang, Eds., in Electrochem. Nucleic Acids Proteins, Towar. Electrochem. Sensors Senomics Proteomics, Elsevier, Amsterdam, The Netherlands, 2005.

[6] J. Barek, A. G. Fogg, A. Muck, J. Zima, Crit. Rev. Anal. Chem. 2001, 31, 291-309.

[7] E. Palecek, Anal. Biochem. 1988, 170, 421-431.

[8] M. Fojta, F. Jelen, L. Havran, E. Palecek, Curr. Anal. Chem. 2008, 4, 250-262.

[9] X. Luo, A. Morrin, A. J. Killard, M. R. Smyth, Electroanalysis 2006, 18, 319-326.

[10] X.-F. Zhang, Z.-G. Liu, W. Shen, S. Gurunathan, Int. J. Mol. Sci. 2016, 17, 1534.

[11] K. S. Siddiqi, A. Husen, R. A. K. Rao, J. Nanobiotechnol 2018, 16, 14. 
[12] Y. ' An Qing, L. Cheng, R. Li, G. Liu, Y. Zhang, X. Tang, J. Wang, H. Liu, Y. Qin, Int. J. Nanomedicine Dovepress 2018, 13, 3311-3327.

[13] P. Juskova, F. Foret, J. Sep. Sci. 2011, 34, 2779-2789.

[14] J. N. Tey, I. P. M. Wijaya, J. Wei, I. Rodriguez, S. G. Mhaisalkar, Microfluid. Nanofluidics 2010, 9, 1185-1214.

[15] P. Juskova, Microfluidics and Nanotechnology for Bioanalytical Applications, Masaryk University Brno, 2013.

[16] B. Yosypchuk, L. Novotny, Crit. Rev. Anal. Chem. 2002, 32, 141-151.

[17] B. Yosypchuk, J. Barek, Crit. Rev. Anal. Chem. 2009, 39, 189-203.

[18] A. Danhel, J. Barek, Curr. Org. Chem. 2011, 15, 2957-2969.

[19] A. Danhel, B. Josypcuk, J. Barek, M. Fojta, Chem. List. 2016, 110, 215-221.

[20] L. Katsikas, M. Gutiérrez, A. Henglein, J. Phys. Chem. 1996, 100, 11203-11206.

[21] A. Henglein, C. Brancewicz, Chem. Mater. 1997, 4756, 2164-2167.

[22] J. T. T. Pang, I. M. Ritchie, Electrochim. Acta 1982, 27, 683-689.

[23] A. Danhel, V. Mansfeldova, P. Janda, V. Vyskocil, J. Barek, Analyst 2011, 136, 3656.

[24] J. Tvrdikova, A. Danhel, J. Barek, V. Vyskocil, Electrochim. Acta 2012, 73, 23-30.

[25] A. Danhel, F. Ligmajer, T. Sikola, A. Walcarius, M. Fojta, J. Electroanal. Chem. 2018, $821,53-59$.

[26] D. Valera, P. J. Espinoza-Montero, J. Alvarado, P. Carrera, P. Bonilla, L. Cumbal, L. Fernández, Sensors Actuators, B Chem. 2017, 253, 1170-1179.

[27] P. Sebest, L. Fojt, V. Ostatna, M. Fojta, A. Danhel, Electrodeposited Silver Amalgam Particles on Pyrolytic Graphite in (Spectro)Electrochemical Detection of 4-nitrophenol, DNA and GFP. Anal. Chem. 2019 (submited).

[28] P. Juskova, V. Ostatna, E. Palecek, F. Foret, Anal. Chem. 2010, 82, 2690-2695.

[29] A. Danhel, K. K. Shiu, B. Yosypchuk, J. Barek, K. Peckova, V. Vyskocil, Electroanalysis 
2009, 21, 303-308.

[30] R. Pfeifer, J. Moreira, M. Nascimento, Int. J. Electrochem. Sci. 2015, 10, 7261-7274.

[31] “4-nitrophenol (US EPA),” https://www.epa.gov, (awailable 5.5.2019).

[32] A. J. Bard, M. Stratmann, Encyclopedia of Electrochemistry: Inorganic Chemistry, John Willey \& Sons, New York, 2006.

[33] F. Ligmajer, M. Horak, T. Sikola, M. Fojta, A. Danhel, J. Phys. Chem. C 2019. (submited)

[33] A. Milchev, W. S. Kruijt, M. Sluyters-Rehbach, J. H. Sluyters, J. Electroanal. Chem. 1993, 350, 89-95.

[34] W. S. Kruijt, J. H. Sluyters, A. Milchev, J. Electroanal. Chem. 1994, 371, 13-26.

[35] Y. D. Gamburg, G. Zangari, Y. D. Gamburg, G. Zangari, in Theory Pract. Met. Electrodepos., Springer New York, New York, NY, 2011, pp. 265-316.

[36] I. S. Krull, M. E. Swartz, LC-GC Eur. 1998, 16, 922-924.

[37] R. Pfeifer, P. Tamiasso-Martinhon, C. Sousa, J. C. Moreira, M. A. C. Do Nascimento, J. Barek, Monatshefte fur Chemie 2016, 147, 111-118.

[38] E. Lukasava, F. Jelen, E. Palecek, Gen. Physiol. Biophys. 1982, 1, 53-70.

[39] L. Havran, M. Fojta, E. Palecek, Bioelectrochemistry 2004, 63, 239-243.

[40] M. Fojta, S. Billova, L. Havran, H. Pivonkova, H. Cernocka, P. Horakova, E. Palecek, Anal. Chem. 2008, 80, 4598-4605.

[41] E. Palecek, M. Trefulka, M. Fojta, Electrochem. commun. 2009, 11, 359-362.

[42] E. Palecek, M. Fojta, Anal. Chem. 2001, 73, 74 A-83 A.

[43] M. Fojta, P. Kostecka, H. Pivonkova, P. Horakova, L. Havran, Curr. Anal. Chem. 2011, 7, 35-50. 